\title{
Association of the location and initial degree of malignant central airway stenosis with the risk of severe restenosis after interventional bronchoscopy
}

\author{
Saibin Wang ${ }^{1 *} \mathbb{0}$, Renzhi Zhou', Siyao Zhu² and Dan Yan ${ }^{1}$
}

\begin{abstract}
Background: Therapeutic bronchoscopy is one of the effective methods in the treatment and management of malignant central airway stenosis (MCAS). However, restenosis after therapeutic bronchoscopy frequently occurs and severe restenosis (SR) can be life-threatening. Therefore, this study aimed at investigating the risk factors for SR after therapeutic bronchoscopy.
\end{abstract}

Methods: The data of 233 consecutive cases with MCAS who were subjected to therapeutic bronchoscopy between 2015 and 2020 at a tertiary hospital were collected. Patients were divided into SR group and non-SR during 6 months after therapeutic bronchoscopy. Multiple logistic regression analysis was performed to determine the risk factors for SR.

Results: SR during 6 months after therapeutic bronchoscopy occurred in 39.5\% (92/233) of patients. The location and the initial degree of MCAS were associated with SR, as assessed by multiple logistic regression analysis $(P<0.05)$. The risk of SR after therapeutic bronchoscopy in the left main bronchus, right main bronchus, and right intermediate bronchus increased, compared to the risk when of MCAS was located in the trachea (OR (95\% Cl) of 8.821 (1.85025.148), 6.583 (1.791-24.189), and 3.350 (0.831-13.511), respectively). In addition, the initial degree of MCAS was positively associated with an increased risk of SR (OR 1.020; 95\% Cl 1.006-1.035).

Conclusions: MCAS located in the left main bronchus, right main bronchus and right intermediate bronchus, as well as the higher initial degree of MCAS were independent risk factors for SR during 6 months after therapeutic bronchoscopy.

Keywords: Malignant central airway stenosis, Restenosis, Bronchoscopy, Lung cancer

\section{Introduction}

Malignant central airway stenosis (MCAS) is referred to the stenosis of the central airway including the trachea, left main bronchus (LMB), right main bronchus (RMB),

\footnotetext{
*Correspondence: saibinwang@hotmail.com

${ }^{1}$ Department of Respiratory Medicine, Jinhua Municipal Central Hospital, No. 365, East Renmin Road, Jinhua 321000, Zhejiang Province, China

Full list of author information is available at the end of the article
}

and right intermediate bronchus (RIB) caused by primary or metastatic malignancies. MCAS commonly leads to different levels of dyspnea and even asphyxia in the patients, which could be frequent outcomes if MCAS is left untreated $[1,2]$. The treatment and management of MCAS were very difficult until the time in which specific airway interventions were available, such as electrocautery, cold ablation, mechanical resection, laser, and airway dilatation [1-5]. MCAS can be rapidly resolved 
in most patients using therapeutic bronchoscopy $[2,6]$. However, airway restenosis commonly occurs after therapeutic bronchoscopy with the progress of the tumor. Therefore, it is important that interventionists know the risk factors and timing of the restenosis in the management and treatment of MCAS. Unfortunately, few reports are available regarding this important aspect.

Hence, a retrospective study was performed to assess severe restenosis (SR) during 6 months after therapeutic bronchoscopy, to identify the independent risk factors for SR in these patients.

\section{Methods}

\section{Study design and data collection}

This was a retrospective study using data collected from consecutive patients who were diagnosed with MCAS and were subjected to therapeutic bronchoscopy at a large-scale tertiary hospital in China between May 2015 and August 2020. The criteria used to decide the first intervention in this study were when central airway obstruction exceeded $50 \%$ and the patients consented to therapeutic bronchoscopy regardless of symptoms. Patients with $100 \%$ central airway obstruction in whom it was not possible to open the obstruction after therapeutic bronchoscopy, or those without postoperative computed tomography (CT) scan during the 6 months follow-up were excluded. The study was approved by the ethics committee of the Jinhua Municipal Central Hospital (Jinhua, China) (No. 2020-LLSC-334). All analyzed data were anonymous, and therefore, the informed consent was waived [7].

The following variables were collected: age, gender, smoking, co-morbidities including hypertension, diabetes, and chronic obstructive pulmonary disease, locations of the lesion (the trachea, LMB, RMB, and RIB), histological type of malignancies (lung adenocarcinoma, lung squamous cell carcinoma, small cell lung cancer and other), dyspnea index (DI) [8], initial degree of MCAS (calculated by the formula: largest area occupied by the lesion in the lumen/total area of the lumen $\times 100 \%$, based on the cross-sectional CT imaging), degree of residual stenosis after therapeutic bronchoscopy, and postbronchoscopy therapies (radiotherapy, chemotherapy, molecule-targeted therapy, and immunotherapy). In this study, SR was defined as the degree of restenosis that exceeds half of the residual stenosis after interventional treatment, otherwise classified as non-SR.

\section{Bronchoscopy procedures}

All patients were subjected to bronchoscopy under general anesthesia and the procedure was performed as previously described [9]. In this study, all the interventional procedure was performed using fiberoptic bronchoscope
(BF-1T60, Olympus Corp., Tokyo, Japan) via a laryngeal mask (Well Lead Medical Co., Ltd., Guangzhou, China). The modalities of the interventional bronchoscopy in this study included electrocautery, argon plasma coagulation, cryotherapy, and airway stent implantation (Nickel-Titanium shape-memory alloy net-like stent (Nanjing Minimally Invasive Medical Technology Co., Ltd., Nanjing, China) or Membrane-covered metallic stent (BSC Int'l Medical Trade (Shanghai) Co., Ltd., Shanghai, China).

\section{Statistical analysis}

Continuous data are presented as mean \pm standard deviation and categorical variables as number and percentage. Unpaired t-tests or Mann-Whitney U test, Pearson chi-squared tests or Fisher's exact test was applied as appropriate to compare the SR with the non-SR group. Variables that yielded a $P$ value $<0.05$ in the univariate analysis between the two groups were included in the multiple logistic regression. In addition, the general additive model was performed after adjusting for potential confounding factors [9] R software (version 3.5.1; R Foundation for Statistical Computing, Vienna, Austria) was used for statistical analysis and a $P$ value $<0.05$ was considered statistically significant.

\section{Results}

The data of 233 MCAS cases treated with interventional bronchoscopy were collected and analyzed in this study. A total of $91.8 \%$ were males and $71.2 \%$ of the population was represented by smokers as shown in Table 1 . The lesions located in the trachea, LMB, RMB, and RIB represented $14.6 \%, 32.2 \%, 33.5 \%$ and $19.7 \%$, respectively. More than half of the patients $(53.6 \%)$ had a DI of grade 4. The proportion of patients with DI of grade 4 in the SR group (57.6\%) was higher than that in the non-SR group (51.1\%), but the difference was not statistically significant (Table 2, $P>0.05$ ). During 6 months after therapeutic bronchoscopy, 92 patients (39.5\%) developed SR (Fig. 1). Patients with SR in this study received an average of 2.1 interventions. In these lesions, the rate of developing SR at 1-month, 2-month, and 3-month after bronchoscopy intervention was $26.1 \%, 65.2 \%$, and $79.3 \%$, respectively. The rate of the different pathological types of tumors such as lung adenocarcinoma, lung squamous cell carcinoma, and lung small cell carcinoma was $2.1 \%, 79.8 \%$ and $7.3 \%$, respectively. In this study, $90 \%$ of patients with primary lung cancer were stage IIIB-IV based on the 8th edition of the TNM classification for lung cancer. A total of $43.8 \%$ of patients received more than two different treatments (chemotherapy, radiotherapy, moleculetargeted therapy, and immunotherapy) after therapeutic bronchoscopy. The overall median survival of the population in this study was 26.3 months. 
Table 1 Baseline characteristics of the study participants

\begin{tabular}{|c|c|}
\hline Characteristics & Value \\
\hline Age $(y),(m e a n \pm S D)$ & $66.6 \pm 8.2$ \\
\hline Male, n (\%) & $214(91.8)$ \\
\hline Smoking, n (\%) & $166(71.2)$ \\
\hline \multicolumn{2}{|l|}{ Coexisting disease, $n(\%)$} \\
\hline COPD & $21(9.0)$ \\
\hline Hypertension & $58(24.9)$ \\
\hline Diabetes & $11(4.7)$ \\
\hline Dyspnea index, n (\%) & $13(2.5)$ \\
\hline 0 & $42(18.0)$ \\
\hline 1 & $9(3.9)$ \\
\hline 2 & $27(11.6)$ \\
\hline 3 & $30(12.9)$ \\
\hline 4 & $125(53.6)$ \\
\hline \multicolumn{2}{|l|}{ Location of the lesion, $n$ (\%) } \\
\hline Trachea & $34(14.6)$ \\
\hline Left main bronchus & $75(32.2)$ \\
\hline Right main bronchus & $78(33.5)$ \\
\hline Right intermediate bronchus & $46(19.7)$ \\
\hline Initial degree of MCAS, (mean \pm SD, \%) & $76.9 \pm 23.9$ \\
\hline Post-intervention residual stenosis, (mean $\pm \mathrm{SD}, \%$ ) & $28.4 \pm 17.3$ \\
\hline \multicolumn{2}{|l|}{ Histological types, $n(\%)$} \\
\hline Lung adenocarcinoma & $5(2.1)$ \\
\hline Lung squamous cell carcinoma & $186(79.8)$ \\
\hline SCLC & $17(7.3)$ \\
\hline Other* & $25(10.7)$ \\
\hline Stent implantation, n (\%) & $56(24.0)$ \\
\hline \multicolumn{2}{|l|}{ Other treatments after intervention, ${ }^{* *} n(\%)$} \\
\hline 0 & $50(21.5)$ \\
\hline 1 & $81(34.8)$ \\
\hline$\geq 2$ & $102(43.8)$ \\
\hline Severe restenosis, n (\%) & $92(39.5)$ \\
\hline
\end{tabular}

COPD chronic obstructive pulmonary disease, MCAS malignant central airway stenosis, SCLC small-cell lung carcinoma

*Other histological types including adenoid cystic carcinoma, lymphoma, large cell neuroendocrine carcinoma, mesenchymal tumor, soft tissue sarcoma, and metastatic malignancies (esophageal cancer, gastric cancer, colorectal adenocarcinoma, and thyroid papillary carcinoma)

**Other treatments including radiotherapy, chemotherapy, molecule-targeted therapy, and immunotherapy. 0 , patients didn't receive any other treatment after therapeutic bronchoscopy; 1 , patients received one other treatment after therapeutic bronchoscopy; $\geq 2$, patients received at least two other treatments after therapeutic bronchoscopy

A total of 56 patients received stent implantation $(21$ with Nickel-Titanium shape-memory alloy net-like stent and 35 with membrane-covered metallic stent). Among patients with lung squamous cell carcinoma, 30.1\% $(43 / 143)$ of the cases received stent implantation. There was no statistically significant difference $(P>0.05)$ in the incidence of SR between the stent implantation group $(37.2 \%)$ and the non-stent implantation group (43.4\%) within 6 months after the intervention. However, the mean residual degree of post-intervention stenosis was lower in the stent implantation group $(22.7 \%)$ than in the non-stent implantation group $(30.1 \%) \quad(P<0.05)$. Among these cases, the fracture of the implanted stent was observed in one case, its migration was observed in two cases, and the intervention-related bleeding exceeding $100 \mathrm{ml}$ was observed in two cases. No death occurred due to bronchoscopy.

The initial degree of MCAS, the degree of post-intervention residual stenosis and the location of MCAS were correlated with SR during 6 months after therapeutic bronchoscopy, as assessed by univariate analysis (Table 2). However, only the initial degree of MCAS and its location were independent risk factors associated with $\mathrm{SR}$, as assessed by multiple logistic regression analysis $(P<0.05$, Table 3). Furthermore, the lesions located in the LMB, RMB, and RIB were more prone to SR after therapeutic bronchoscopy compared to those located in the trachea, with an odds ratio [OR] (95\% confidence interval [CI]) of 8.821 (1.850-25.148), 6.583 (1.791-24.189), and 3.350 (0.831-13.511), respectively. However, no significant difference in the incidence of SR was found among LMB, RMB, and RIB ( $P>0.05$, Fig. 2$)$. The initial degree of MCAS was positively associated with the risk of SR during 6 months after therapeutic bronchoscopy ( $P$ for trend $=0.025$, Table 3; Fig. 3).

\section{Discussion}

The present study revealed that the location of the MCAS and its initial degree were associated with SR during 6 months after therapeutic bronchoscopy. Specifically, MCAS located in the LMB, RMB, and RIB was more prone to SR after therapeutic bronchoscopy compared to those found in the trachea. In addition, the higher the initial degree of MCAS, the greater the risk of SR after therapeutic bronchoscopy.

Central airway stenosis or obstruction is a common clinical chest disorder, and it is also a difficult clinical problem for clinicians. MCAS has become increasingly frequent due to the high incidence of malignancies, especially lung cancer $[2,10]$. Indeed, $20-30 \%$ of lung cancer patients develop complications related to airway obstruction [2]. Patients commonly die of asphyxiation resulting from severe airway stenosis. Fortunately, the rapid development of interventional bronchoscopy (e.g., electrocautery, cryotherapy, balloon bronchoplasty and airway stent implantation) provides an efficient solution for airway stenosis $[1,2,4,11,12]$. Most of the MCAS can be ameliorated using therapeutic bronchoscopy, thus patients can be quickly relieved $[2,13]$. Interventional bronchoscopy has a significant effect on symptom relief and quality of life improvement, and it is generally considered as 
Table 2 Univariable analysis of potential risk factors for severe restenosis after therapeutic bronchoscopy

\begin{tabular}{|c|c|c|c|}
\hline \multirow[t]{2}{*}{ Variables } & \multicolumn{2}{|c|}{ Severe restenosis } & \multirow[t]{2}{*}{$P$ value } \\
\hline & No $(n=141)$ & Yes $(n=92)$ & \\
\hline Age $(y),($ mean $\pm S D)$ & $65.9 \pm 8.7$ & $67.6 \pm 7.3$ & 0.123 \\
\hline \multicolumn{4}{|l|}{ Gender, $n(\%)$} \\
\hline Male & $126(89.4)$ & 88 (95.7) & \multirow[t]{2}{*}{0.086} \\
\hline Female & $15(10.6)$ & $4(4.3)$ & \\
\hline \multicolumn{4}{|l|}{ Diabetes, n (\%) } \\
\hline Yes & $7(5.0)$ & $4(4.3)$ & \multirow[t]{2}{*}{0.828} \\
\hline No & $134(95.0)$ & $88(95.7)$ & \\
\hline \multicolumn{4}{|l|}{ Hypertension, n (\%) } \\
\hline Yes & $39(27.7)$ & $19(20.7)$ & \multirow[t]{2}{*}{0.227} \\
\hline No & $102(72.3)$ & $73(79.3)$ & \\
\hline \multicolumn{4}{|l|}{ COPD, $n(\%)$} \\
\hline Yes & $13(9.2)$ & $8(8.7)$ & \multirow[t]{2}{*}{0.891} \\
\hline No & $128(90.8)$ & $84(91.3)$ & \\
\hline \multicolumn{3}{|l|}{ Dyspnea index, n (\%) } & \multirow[t]{6}{*}{0.618} \\
\hline 0 & $26(18.4)$ & $16(17.4)$ & \\
\hline 1 & $5(3.5)$ & $4(4.3)$ & \\
\hline 2 & $16(11.3)$ & $11(12.0)$ & \\
\hline 3 & $22(15.6)$ & $8(8.7)$ & \\
\hline 4 & $72(51.1)$ & $53(57.6)$ & \\
\hline Initial degree of MCAS, (mean \pm SD, \%) & $72.3 \pm 25.0$ & $83.9 \pm 20.4$ & $<0.001$ \\
\hline Post-intervention residual stenosis, (mean \pm SD, \%) & $26.1 \pm 17.8$ & $31.8 \pm 15.9$ & 0.012 \\
\hline \multicolumn{3}{|l|}{ Location of the lesion, $n(\%)$} & \multirow[t]{5}{*}{$<0.001$} \\
\hline Trachea & $31(22.0)$ & $3(3.3)$ & \\
\hline Left main bronchus & $39(27.7)$ & $36(39.1)$ & \\
\hline Right main bronchus & $41(29.1)$ & $37(40.2)$ & \\
\hline Right intermediate bronchus & $30(21.3)$ & $16(17.4)$ & \\
\hline \multicolumn{3}{|l|}{ Histological types, $n$ (\%) } & \multirow[t]{5}{*}{0.463} \\
\hline Lung adenocarcinoma & $4(2.8)$ & $1(1.1)$ & \\
\hline Lung squamous cell carcinoma & $108(76.6)$ & $78(84.8)$ & \\
\hline SCLC & $12(8.5)$ & $5(5.4)$ & \\
\hline Other* & $17(12.1)$ & $8(8.7)$ & \\
\hline \multicolumn{3}{|l|}{ Other treatments after intervention, ${ }^{* *} n(\%)$} & \multirow[t]{4}{*}{0.366} \\
\hline 0 & $34(24.1)$ & $16(17.4)$ & \\
\hline 1 & $45(31.9)$ & $36(39.1)$ & \\
\hline$\geq 2$ & $62(44.0)$ & $40(43.5)$ & \\
\hline
\end{tabular}

COPD chronic obstructive pulmonary disease, MCAS malignant central airway stenosis, SCLC small-cell lung carcinoma

*Other histological types including adenoid cystic carcinoma, lymphoma, large cell neuroendocrine carcinoma, mesenchymal tumor, soft tissue sarcoma, and metastatic malignancies (esophageal cancer, gastric cancer, colorectal adenocarcinoma, and thyroid papillary carcinoma)

**Other treatments including radiotherapy, chemotherapy, molecule-targeted therapy, and immunotherapy. 0 , patients didn't receive any other treatment after therapeutic bronchoscopy; 1 , patients received one other treatment after therapeutic bronchoscopy; $\geq 2$, patients received at least two other treatments after therapeutic bronchoscopy

a palliative treatment [14-16]. However, importantly, a recent study (EVERMORE trial) showed that interventional bronchoscopy, as a part of an integrated treatment, improved 1-year survival in patients with locally advanced non-small cell lung cancer (stage IIIB) and associated central airways obstruction [17]. Moreover, genetic and anatomic phenotyping showed the potential to identify patients who may gain life expectancy from interventional bronchoscopy [17]. Actually, airway stenosis or obstruction reoccurs frequently with the progress of the tumor. Therefore, most of the patients are inevitably subjected to bronchoscopy treatment again or 

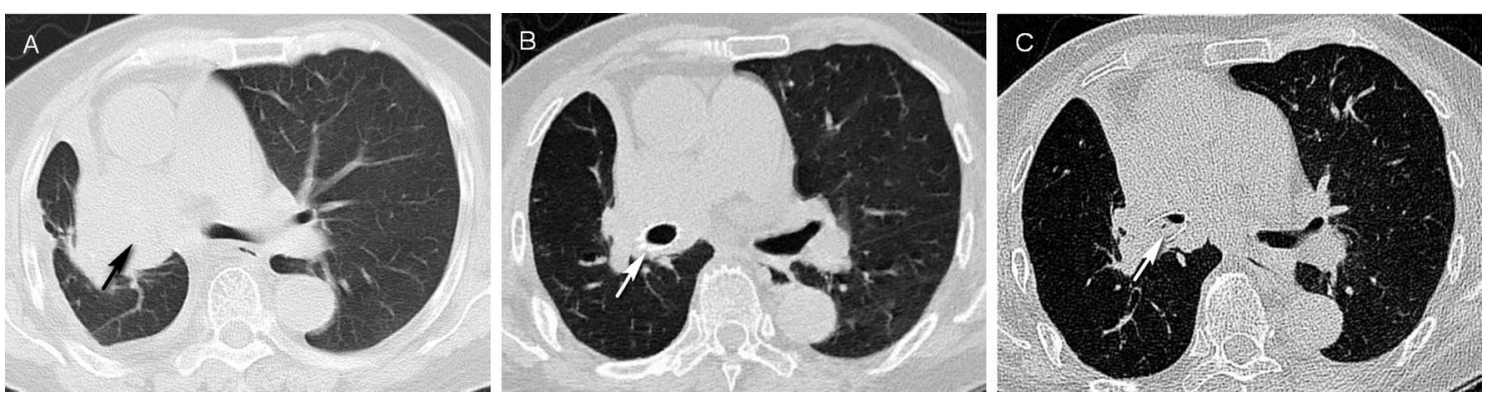

Fig. 1 Severe restenosis of MCAS after therapeutic bronchoscopy. a The right main bronchus was completely obstructed by the lesion on the cross-sectional CT scan (black arrow). b Airway stent implantation improved the stenosis of the right main bronchus on CT scan (arrow). c Severe restenosis of the right main bronchus was observed 3 months after stent implantation on CT scan (arrow). MCAS malignant central airway stenosis

Table 3 Multivariable analysis of risk factors for severe restenosis after therapeutic bronchoscopy

\begin{tabular}{llll}
\hline Variables & Odds ratio & $\mathbf{9 5 \%} \mathbf{C l}$ & $\boldsymbol{p}$ value \\
\hline $\begin{array}{l}\text { Initial degree of MCAS } \\
\text { Initial degree of MCAS tertile }\end{array}$ & 1.020 & $1.006-1.035$ & 0.005 \\
$\quad$ Low & 1.0 & 1.0 & \\
$\quad$ Middle & 2.637 & $1.179-5.897$ & 0.018 \\
$\quad$ High & 2.568 & $1.178-5.596$ & 0.018 \\
$\begin{array}{l}\text { P for trend } \\
\text { Post-intervention residual stenosis }\end{array}$ & 1.003 & $0.985-1.022$ & 0.748 \\
$\begin{array}{l}\text { Location of thelesion } \\
\quad \text { Trachea }\end{array}$ & 1.0 & 1.0 & \\
$\quad$ Left main bronchus & 8.821 & $1.850-25.148$ & 0.004 \\
$\quad$ Right main bronchus & 6.583 & $1.791-24.189$ & 0.005 \\
$\quad$ Right intermediate bronchus & 3.350 & $0.831-13.511$ & 0.089 \\
\hline
\end{tabular}

MCAS malignant central airway stenosis

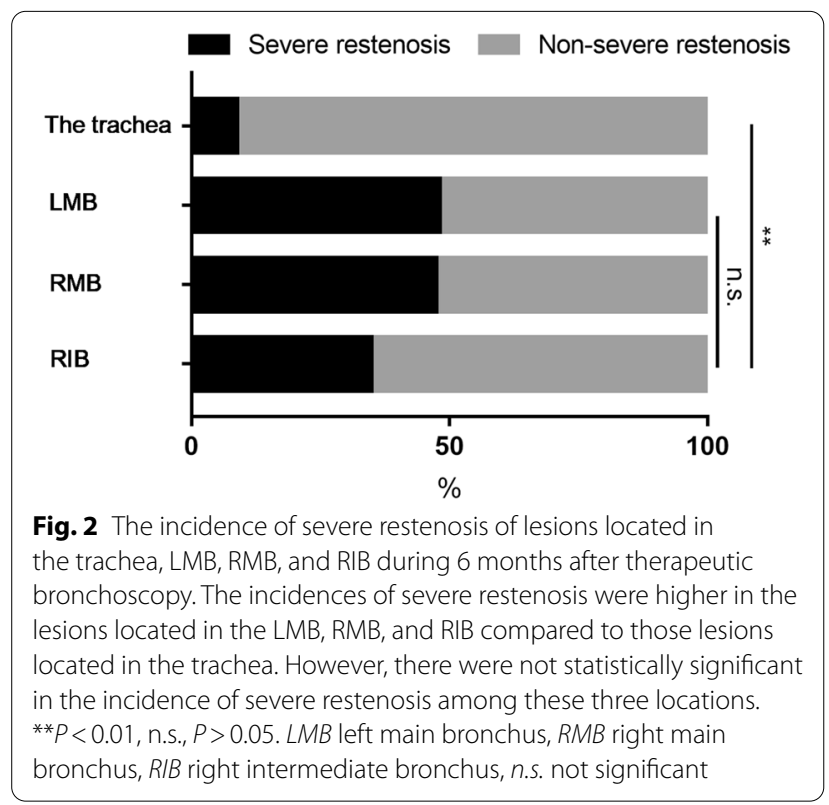

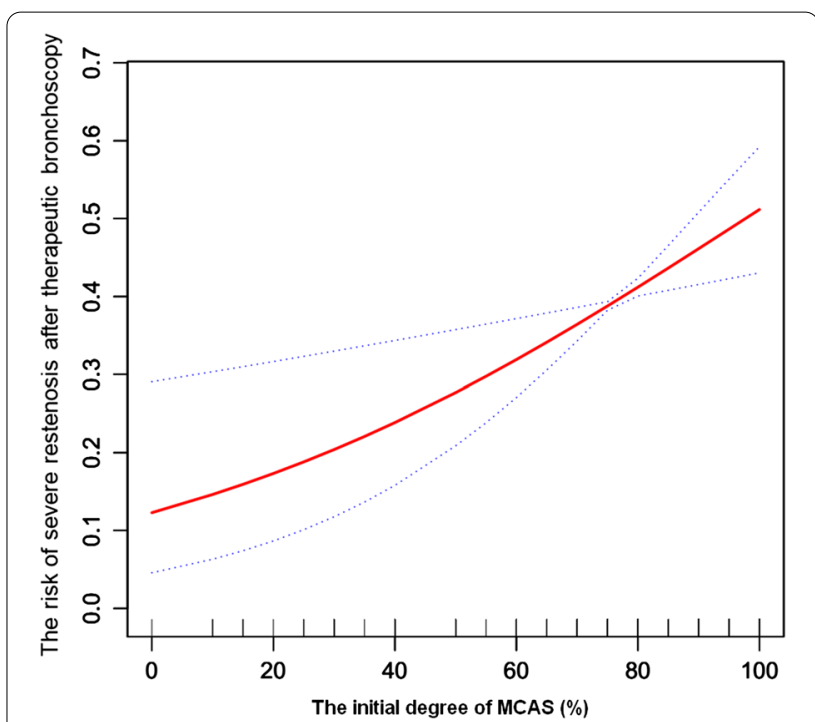

Fig. 3 The relationship between the initial degree of MCAS and the risk of severe restenosis of MCAS during 6 months after therapeutic bronchoscopy. Adjusted variables: age, the location of the lesion, histological type of the lesion, stent implantation and other treatments after intervention. MCAS malignant central airway stenosis

even more times. Thus, clinicians need to know how long it takes for severe, life-threatening airway restenosis to occur after the first bronchoscopic treatment, and what the risk factors for SR are. However, as far as we know, few reports are available focusing on this issue.

The cause of MCAS included primary and metastatic malignant tumors [18]. In our study, $79.8 \%$ of the patients with MCAS were affected by lung squamous cell carcinoma, in agreement with a previous report [19]. Metastatic tumors in the central airway mainly derive from digestive tract tumors, blood tumors and thyroid tumors [1]. As regards the incidence of restenosis of the central airway during 6 months after interventional bronchoscopy, Luo et al. reported that its rate was $10.9-30.7 \%$ 
after anti-tuberculosis therapy combined with interventional bronchoscopy in 152 patients with endobronchial tuberculosis [20]. However, few reports on malignant airway restenosis are available to date. In the present study, severe dyspnea (DI of grade 4) due to MCAS was observed in more than half of the patients, with this phenomenon appearing to be higher in the SR group, but it is notable that more than half of the patients in the nonSR group still had severe dyspnea. The reason why the DI did not reach a statistically significant difference between the two groups might be related to the small sample size and the large degree of post-intervention residual stenosis (Table 2). This suggests that initial treatment with adequate airway opening is crucial for the relief of the patient's symptoms. Our study revealed that the 6-month incidence of SR reached 39.5\%. Among these SR patients, SR occurred at 1-month in more than onequarter $(26.1 \%)$ of patient, at 2-month in more than onehalf (65.2\%), and at 3-month in more than three-quarters (79.3\%). This result might provide the answer regarding the more appropriate timing for the next interventional therapy to cure MCAS.

As regards the risk factors for SR, our study showed that the location of the lesion was associated with the risk of SR after therapeutic bronchoscopy. The lesions located in the trachea were less frequent occurrence of postoperative SR than those in the mainstem bronchi (LMB, RMB, and RIB), indicating that the severity of airway restenosis might be related with the diameter of the airway. Moreover, our results revealed that the initial degree of MCAS was an independent risk factor for postoperative SR. The more severe the initial stenosis, the greater the risk of restenosis occurrence. The relationship between them was strong with both no adjustment and adjustment for potential confounders (age, the location of the lesion, histological type of the lesion, stent implantation and other postoperative treatments). Although the underlying mechanism needs to be further elucidated, our hypothesis was that this might be related to the rate of tumor progression. In addition, granulation tissue hyperplasia is the second frequent complication 6 months after stent placement [21]. Our analysis revealed that both in the overall study population and patients with lung squamous cell carcinoma, the implantation of the airway stent contributed to a lower degree of restenosis, but failed to reduce the incidence of SR as compared to those patients without stent implantation within 6 months after therapeutic bronchoscopy.

This study presents several limitations. Firstly, since this study was a single-center retrospective analysis, it suffered from potential unmeasured confounders and bias despite stringent statistical adjustments were performed to minimize residual confounding factors; therefore prospective investigations are needed to verify our results. Secondly, all interventions in the collected cases were performed by two bronchoscopists with more than 10-year experience, and the interventional modalities were relatively the same, but it was not possible to perform the same intervention method for all lesions, and different intervention methods might result in different effects on the formation of airway restenosis. Thirdly, a certain delay might be present between the time when the patient was diagnosed with SR by postoperative CT scan and the time when SR actually occurred. Therefore, the time to SR occurrence might be underestimated in our study; in other words, the time of SR was probably earlier than the time that was observed. Fourthly, because the procedure of MCAS in this study was carried out with fiberoptic bronchoscope via laryngeal mask, the results of the study cannot be extrapolated to other interventions, such as rigid bronchoscopy, which could be more effective in removing central airway cancer tissues. Additionally, considering the different timing of intervention in the course of the disease, and the different stage, histological type and biology of the cancers analyzed in the present study, it was not appropriate to carry out survival analysis for this study population. Nevertheless, despite the aforementioned potential limitations, our study was the first revealing independent risk factors and the time of formation of postoperative SR in the population affected by MCAS.

\section{Conclusions}

Patients with MCAS located in the LMB, RMB, and RIB had a high risk of SR during 6 months after therapeutic bronchoscopy. In addition, patients with a higher initial degree of MCAS showed an increased risk of postoperative SR. The results of our research might help clinicians in the assessment and management of patients with MCAS. The knowledge of the risk factors of restenosis and the choice of the appropriate time for airway interventional therapy could improve patient's quality of life and survival.

\section{Abbreviations \\ MCAS: malignant central airway stenosis; SR: Severe restenosis; OR: Odds ratio; $\mathrm{Cl}$ : Confidence interval.}

\section{Acknowledgements}

We appreciate the help and support of all the participants involved in the study.

\section{Authors' contributions}

SW contributed substantially to the study design, data analysis and interpretation, the writing of the manuscript, and takes responsibility for the integrity of the data and the accuracy of the data analysis. RZ, SZ and DY contributed to data collection and data interpretation. All authors read and approved the final manuscript. 


\section{Funding}

This study was supported by the Medical and Health Science and Technology Plan Project of Zhejiang Province (No. 2018RC079, 2022495999), Youth Research Fund of Jinhua Municipal Central Hospital (No. JY2017-2-05), and the Science and Technology Project of Jinhua City (No. 2020XG-15). Zhejiang health and Health Committee, Jinhua Municipal Central Hospital and Jinhua Science and Technology Bureau provided funding but had no role in study design or collection, analysis or interpretation, nor in writing of the manuscript.

\section{Availability of data and materials}

The datasets used and/or analyzed during the current study are available from the corresponding author on reasonable request.

\section{Declarations}

\section{Ethics approval and consent to participate}

The study was approved by the ethics committee of the Jinhua Municipal Central Hospital (Jinhua, China) (No. 2020-LLSC-334). Written informed consent was waived by the ethics committee of the Jinhua Municipal Central Hospital.

\section{Consent for publication}

Not applicable.

\section{Competing interests}

The authors declare that they have no competing interests.

\section{Author details}

${ }^{1}$ Department of Respiratory Medicine, Jinhua Municipal Central Hospital, No. 365, East Renmin Road, Jinhua 321000, Zhejiang Province, China. ${ }^{2}$ Shaoxing University School of Medicine, Shaoxing 312000, Zhejiang Province, China.

Received: 21 May 2021 Accepted: 30 September 2021

Published online: 18 October 2021

\section{References}

1. Jin F, Li Q, Li S, et al. Interventional bronchoscopy for the treatment of malignant central airway stenosis: an expert recommendation for China. Respiration. 2019;97(5):484-94.

2. Ernst A, Feller-Kopman D, Becker HD, Mehta AC. Central airway obstruction. Am J Respir Crit Care Med. 2004;169(12):1278-97.

3. Mohan A, Shrestha P, Madan K, et al. A prospective outcome assessment after bronchoscopic interventions for malignant central airway obstruction. J Bronchol Interv Pulmonol. 2020;27(2):95-105.

4. Mudambi L, Miller R, Eapen GA. Malignant central airway obstruction. J Thorac Dis. 2017;9(Suppl 10):S1087-S1110.

5. Miyazawa T, Yamakido M, Ikeda S, et al. Implantation of ultraflex nitinol stents in malignant tracheobronchial stenoses. Chest. 2000;118(4):959-65.
6. Usuda K, Iwai S, Yamagata A, et al. Clinical outcomes and survival following placement of self-expandable metallic stents for central airway stenosis and fistula. Thorac Cancer. 2021;12(1):48-56.

7. Fong M, Braun KL, Chang RM. Native Hawaiian preferences for informed consent and disclosure of results from genetic research. J Cancer Educ. 2006;21(1 Suppl):S47-S52.

8. Tie K, Buckmire RA, Shah RN. The role of spirometry and dyspnea index in the management of subglottic stenosis. Laryngoscope. 2020;130(12):2760-6.

9. Wang S, Zhang J, Lu X. Non-linear association of plasma level of highdensity lipoprotein cholesterol with endobronchial biopsy bleeding in patients with lung cancer. Lipids Health Dis. 2019;18(1):17.

10. Ayers ML, Beamis JF Jr. Rigid bronchoscopy in the twenty-first century. Clin Chest Med. 2001;22(2):355-64.

11. Puchalski J, Musani Al. Tracheobronchial stenosis: causes and advances in management. Clin Chest Med. 2013;34(3):557-67.

12. Lund ME, Garland R, Ernst A. Airway stenting: applications and practice management considerations. Chest. 2007;131(2):579-87.

13. Hautmann H, Gamarra F, Pfeifer KJ, Huber RM. Fiberoptic bronchoscopic balloon dilatation in malignant tracheobronchial disease: indications and results. Chest. 2001;120(1):43-9.

14. Kalsi HS, Thakrar R, Gosling AF, Shaefi S, Navani N. Interventional pulmonology: a brave new world. Thorac Surg Clin. 2020;30(3):321-38.

15. Stephens KE Jr, Wood DE. Bronchoscopic management of central airway obstruction. J Thorac Cardiovasc Surg. 2000;119(2):289-96.

16. Mehta AC, Lee FY, Cordasco EM, Kirby T, Eliachar I, De Boer G. Concentric tracheal and subglottic stenosis. Management using the Nd-YAG laser for mucosal sparing followed by gentle dilatation. Chest. 1993;104(3):673-7.

17. Marchioni A, Andrisani $D$, Tonelli $R$, et al. Integrated interventional bronchoscopy in the treatment of locally advanced non-small lung cancer with central malignant airway obstructions: a multicentric retrospective study (EVERMORE). Lung Cancer. 2020;148:40-7.

18. Iyoda A, Azuma Y, Sano A, et al. Contributions of airway stent for longterm outcome in patients with malignant central airway stenosis or obstruction. J Bronchol Interv Pulmonol. 2021. https://doi.org/10.1097/ LBR.0000000000000749.

19. Cavaliere S, Foccoli P, Farina PL. Nd:YAG laser bronchoscopy. A fiveyear experience with 1,396 applications in 1,000 patients. Chest. 1988;94(1):15-21.

20. Luo LZ, Luo L, Lu ZB, et al. The efficacy of balloon dilatation in clinical improving period for patients who suffered from actively caseating endobronchial tuberculosis and central airway stenosis. Zhonghua Jie He He Hu Xi Za Zhi. 2021;44(3):237-42. [Article in Chinese].

21. Ortiz-Comino RM, Morales A, López-Lisbona R, et al. Silicone stent versus fully covered metallic stent in malignant central airway stenosis. Ann Thorac Surg. 2021;111(1):283-9.

\section{Publisher's Note}

Springer Nature remains neutral with regard to jurisdictional claims in published maps and institutional affiliations.

Ready to submit your research? Choose BMC and benefit from

- fast, convenient online submission

- thorough peer review by experienced researchers in your field

- rapid publication on acceptance

- support for research data, including large and complex data types

- gold Open Access which fosters wider collaboration and increased citations

- maximum visibility for your research: over $100 \mathrm{M}$ website views per year

At BMC, research is always in progress.

Learn more biomedcentral.com/submissions 